\title{
Carcinosarcoma of the Gallbladder Arising in a Patient with Pancreaticobiliary Maljunction: A Case Report and Review of the Literature
}

Kirstin Coetzee ${ }^{1}$, Jones Omoshoro-Jones ${ }^{2}$ and Pam Michelow ${ }^{3 *}$

${ }^{1}$ Registrar, Anatomical Pathology Department, National Health Laboratory Service and University of the Witwatersrand, Johannesburg, South Africa ${ }^{2}$ Senior Specialist Surgeon and Senior Lecturer, Hepatopancreatobiliary Surgery, Department of Surgery, Chris Hani Baragwanath Hospital, University of the Witwatersrand, Johannesburg, South Africa

${ }^{3}$ Principle Medical Officer, Cytology Unit, Anatomical Pathology Department, National Health Laboratory Service and University of the Witwatersrand, Johannesburg, South Africa

\begin{abstract}
Background: Carcinosarcoma of the gallbladder is a very uncommon malignancy. We present a case of gallbladder carcinosarcoma arising in a 38-year-old female patient with pancreaticobiliary maljunction where FNA documented recurrence of this very unusual tumor.

Case: A 38-year old woman presented with signs and symptoms of acute pancreatitis due to gallstones (biliary pancreatitis) Further investigations, including abdominal ultrasonography and endoscopic retrograde cholangiopancreatography (ERCP), revealed an ill-defined mass in the gallbladder, in addition to pancreaticobiliary maljunction. At laparoscopic cholecystectomy, a mass was found within the gallbladder lumen itself. A biphasic malignant neoplasm was diagnosed in a free lying, polypoid mass within the gallbladder lumen. This carcinosarcoma was considered to have been locally confined to the gallbladder, equivalent to a stage T1a, and further surgery was not undertaken. The patient re-presented under a year later with dyspnoea; and a large subdiaphragmatic mass was noted that underwent ultrasound-guided fine needle aspiration (FNA) biopsy. The cytomorphologic findings showed a tumor comprising malignant glandular structures and pleomorphic stromal cells in a metachromatic background, compatible with the previous diagnosis of carcinosarcoma.

Conclusion: Carcinosarcomas are uncommon malignancies and can arise in almost any site where carcinomas arise. Careful attention to morphology will allow the biphasic nature of this lesion to be determined but ancillary investigations may be required.

Carcinosarcoma is a biphasic malignant neoplasm with both malignant epithelial (carcinomatous) and stromal (sarcomatous) elements. Carcinosarcoma occurring in the gallbladder is a rare malignancy of which there have been fewer than seventy reports in the worldwide literature to date, with thirty two of these in the English literature [1-14]. We present a case of gallbladder carcinosarcoma in a young patient with pancreaticobiliary maljunction, treated with cholecystectomy, with subsequent local recurrence diagnosed on image-guided fine needle aspirate (FNA). There appears to be only one other reported case of FNA of a carcinosarcoma of the gallbladder in which an initial diagnosis of a high-grade spindle cell sarcoma was made cytologically [14].
\end{abstract}

Keywords: Gallbladder malignancy; Gallbladder carcinosarcoma; Pancreaticobiliary maljunction

\section{Case Report}

A 38-year-old woman presented in April 2009 with signs and symptoms of biliary pancreatitis (acute abdominal pain, elevated serum amylase and lipase and obstructive jaundice). Her HIV status was negative. On abdominal ultrasonography, there was an ill-defined mass in the gallbladder, and it was uncertain whether this represented bile sludge or a gallbladder tumor. The ERCP findings in our case showed a relatively 'normal' common channel length but the angle of the pancreaticobiliary junction was widened, a congenital biliary malformation within the spectrum of pancreaticobiliary maljunction (PBM) (Figure 1). There were no biliary stones or strictures on ERCP nor biliary duct dilatation suggestive of choledochal cyst. Contrast-enhanced triple phase computed tomography (CT) scan of the abdomen showed neither gallbladder mass nor tumour and the gallbladder and biliary tree were judged to be within acceptable parameters. At subsequent laparoscopic cholecystectomy, dissection was quite demanding due to much fibrosis and inflammation involving the gallbladder and Calot's triangle. The cystic duct was noted to be short and widened. On extraction and opening, the thick-walled, fibrosed gallbladder contained a mass described by the surgeons as a "mucocoele-like lesion".

\section{Histologic findings}

The gallbladder and separate mass were received for histologic evaluation; the mass was in two pieces, the larger of these measuring $82 \times 55 \times 35 \mathrm{~mm}$ and the smaller $65 \times 35 \times 25 \mathrm{~mm}$. Macroscopically, the free lying gallbladder mass comprised a friable, bile-stained tumor

${ }^{*}$ Corresponding author: Pamela Michelow, Principle Medical Officer, Cytology Unit, Anatomical Pathology Department, National Health Laboratory Service and University of the Witwatersrand, Johannesburg, South Africa, Tel: +27114899408; Fax:+27114899411; E-mail: pamela.michelow@nhls.ac.za

Received February 19, 2011; Accepted March 28, 2011; Published March 30 2011

Citation: Coetzee K, Omoshoro-Jones J, Michelow P (2011) Carcinosarcoma of the Gallbladder Arising in a Patient with Pancreaticobiliary Maljunction: A Case Report and Review of the Literature. J Cytol Histol 2:115. doi:10.4172/21577099.1000115

Copyright: (c) 2011 Coetzee $\mathrm{K}$, et al. This is an open-access article distributed under the terms of the Creative Commons Attribution License, which permits unrestricted use, distribution, and reproduction in any medium, provided the original author and source are credited. 
with a necrotic cut surface within which cystic spaces containing brown mucoid material were identified, the largest of which had a diameter of $10 \mathrm{~mm}$. The separate, surgically-incised gallbladder measured 105x60x30 mm and exhibited a thickened wall measuring up to $8 \mathrm{~mm}$ on gross examination. There was extensive ulceration of the mucosal surface. The previous point of attachment of the mass was not apparent, and there were no gallstones or other contents. Histological examination of the tumor confirmed the presence of a polypoid portion of tissue that was, for the most part, non-viable, exhibiting extensive necrosis and infarction. Malignant glands were seen in association with pleomorphic and atypical stromal cells that showed a myxoid and oedematous background with a chondroid appearance in places. The glands showed a serrated architecture with retained formation of lumina and were lined by malignant epithelial cells showing a high degree of nuclear pleomorphism, increased nuclear-cytoplasmic ratio, in addition to abundant and abnormal mitotic activity. The spindle cells of the intervening stroma showed features of malignancy, including enlarged and hyperchromatic nuclei with prominent nucleoli. Abnormal stromal mitoses were seen. There was focal evidence of heterologous differentiation of the stroma: focal osteoid was present which showed calcification and frank bone formation (Figures 2 and 3).

Sections of the gallbladder confirmed a thickened, fibrotic wall within which there was mild chronic inflammation. The gallbladder mucosa was denuded and ulcerated throughout large areas, and there was only partial representation of the gallbladder mucosa despite extensive sampling. Where present, the gallbladder mucosa showed evidence of focal high-grade dysplasia within micropapillary mucosal projections equivalent to adenocarcinoma in-situ. There was no

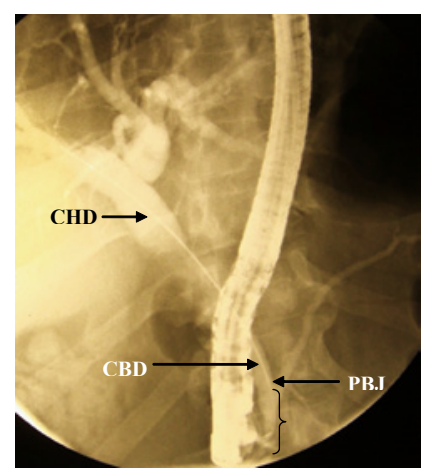

Figure 1: ERCP revealing PBJ pancreatico-biliary junction, $\mathrm{CBD}$ common bile duct, CHD common hepatic duct.

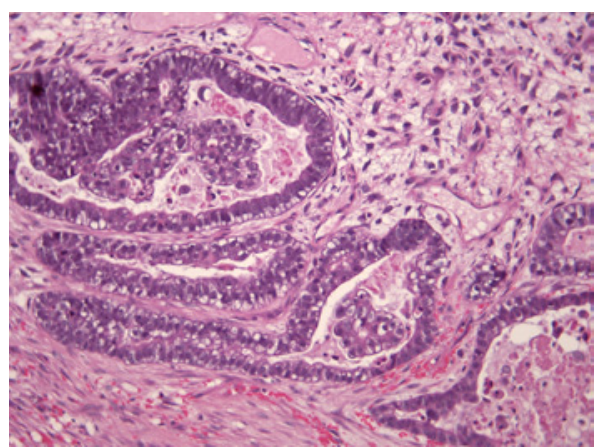

Figure 2: Histology of malignant glandular and stromal components within the gallbladder mass (H-E stain, $x$ 400).

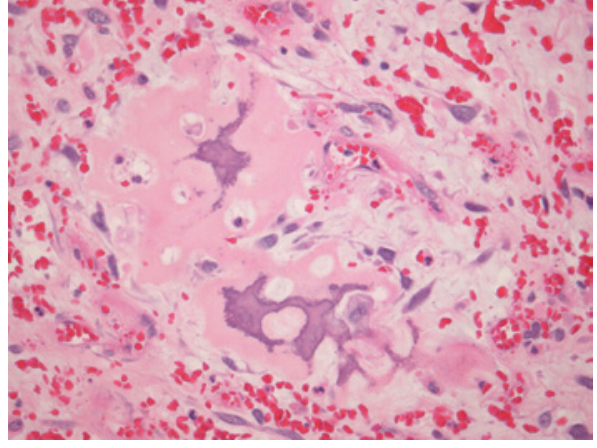

Figure 3: Histology of chondroid differentiation within the carcinosarcoma (H-E stain, $x$ 400).

evidence of invasive malignancy within any of several sections of the gallbladder examined. Immunohistochemical stains performed on the gallbladder showed pancytokeratin $\mathrm{AE} 1 / 3$ positivity in the dysplastic lining epithelium. Smooth muscle markers (desmin, MSA, SMA) failed to highlight atypical stromal cells within the gallbladder wall, and hence it was determined that there was neither morphological nor immunohistochemical evidence of an invasive component, either carcinomatous or sarcomatous, within the gallbladder wall itself.

The final histopathological diagnosis was of a gallbladder carcinosarcoma which was postulated to have arisen either within a gallbladder polyp or as a polypoid tumour of the gallbladder, which had twisted on its pedicle and underwent torsion, infarction and subsequent detachment, thus accounting for its presence as a free lying mass within the gallbladder lumen. Whilst the gallbladder showed focal high-grade dysplasia equivalent to adenocarcinoma in-situ, there was no evidence of invasive carcinoma or carcinosarcoma of the gallbladder itself. Given the apparent local confinement of the malignancy and the absence of other abnormalities of the biliary tree, a policy of watchful follow-up was decided upon by the hepatopancreatobiliary clinicians.

Unfortunately, the patient was lost to follow-up until representation nine months later with dyspnoea and recurrent obstructive jaundice requiring biliary drainage. A large mass under the diaphragm inhibiting respiration was found. Ultrasound-guided FNA of this mass was undertaken.

\section{Cytologic findings}

On cytomorphology, the biphasic nature of the tumor was evident, although the stromal component predominated. The stromal areas consisted of single cells and loosely cohesive clusters in a metachromatic background. The cells were oval in shape with small amounts of wispy cytoplasm and high nuclear-cytoplasmic ratio. The nuclei were pleomorphic in shape and size and varied from round to oval to spindled with irregular nuclear membranes, hyperchromatic and irregularly distributed chromatin and prominent nucleoli. There was no evidence of osteoid formation on FNA. The epithelial component showed fairly well-cohesed groups of cells in glandular arrangements. The cells were cuboidal in shape with eccentrically situated nuclei and eosinophilic cytoplasm. The nuclei were round to oval in shape with coarse chromatin, parachromatin clearing and prominent nucleoli (Figure 4). On immunocytochemistry, the glandular component proved positive for AE1/AE3 while the stromal cells showed MSA positivity (Figures 5 and 6). In some areas, the glandular and stromal components were lying separately while in other sections of the slide, both elements were found together. The patient is currently undergoing palliative chemotherapy under the medical oncology team. 
Citation: Coetzee K, Omoshoro-Jones J, Michelow P (2011) Carcinosarcoma of the Gallbladder Arising in a Patient with Pancreaticobiliary Maljunction: A Case Report and Review of the Literature. J Cytol Histol 2:115. doi:10.4172/2157-7099.1000115

\section{Discussion}

Carcinosarcoma is defined as a malignant neoplasm with both malignant mesenchymal (sarcomatous) and epithelial (carcinomatous) components. Whilst carcinosarcoma under its various, and sometimes site-specific pseudonyms, such as "sarcomatoid carcinoma", "spindle cell carcinoma", "pseudosarcoma", "undifferentiated carcinoma, sarcomatoid type", and "malignant mixed (müllerian) tumour" (female genital tract) occurs with slightly higher frequency in other visceral sites, it is a rare amongst gallbladder neoplasms. To the best of our knowledge, there are less than 70 cases of gallbladder carcinosarcoma which have been reported in the international literature to date, and only 32 of these in the English literature. Most of these cases were described in elderly patients, above the age of 60 years, with an age range of 45 to 90 years, the majority of these being women [1-14]. Our case occurred in a 38 -year-old female patient, which is the youngest

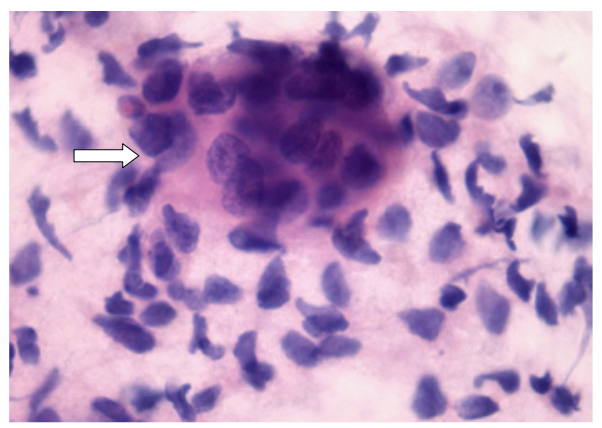

Figure 4: Cytomorphology consisting of malignant glandular cells ( at arrow) within malignant stromal cells (Papanicolaou stain, $x$ 600).

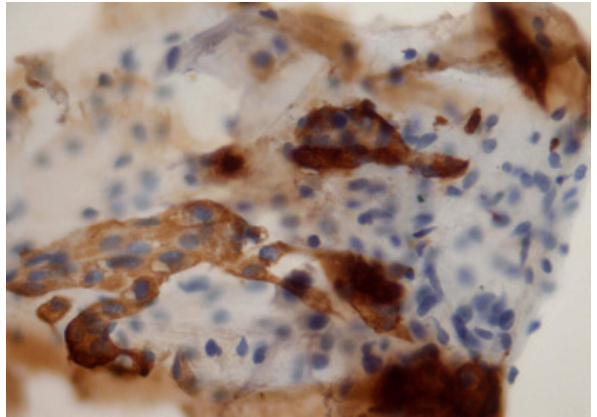

Figure 5: Glandular cells showing positive AE1/AE3 cytoplasmic staining on the FNA specimen while the stromal areas remain unstained (x 600).

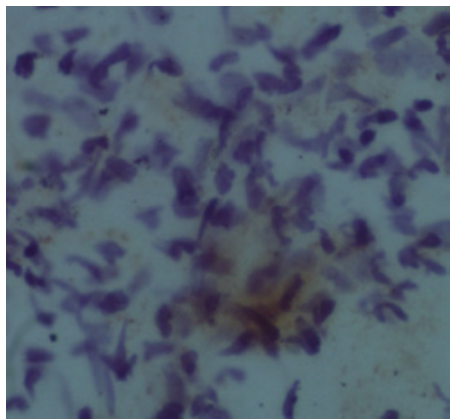

Figure 6: Pleomorphic stromal cells showing positive MSA cytosplasmic staining on the FNA specimen (x 600). reported case in the literature to date. Her presentation at a much younger age may be attributed to the ERCP finding of pancreaticobiliary maljunction (PBM), an entity which has a proven association with biliary carcinogenesis [14-16]. There has been one previous report of gallbladder carcinosarcoma in a patient with "multiple anomalies", including preduodenal portal vein, absence of the pancreatic tail and intestinal malrotation [8], but specific occurrence of carcinosarcoma in a patient with PBM has not yet been described. In 1986, diagnostic criteria established by the Japanese Study Group on Pancreaticobiliary Maljunction (JSPBM) defined the entity by radiological evidence of location of the junction of pancreatic and bile ducts outside of the duodenal wall [13]. This anomalous ductal union creates a so-called "long common channel" or an abnormal angle of ductal union, in which the preventative function of the sphincter of Oddi in mixture of bile and pancreatic juices is absent. Hydrostatic pressure gradients between the pancreatic and biliary ductal systems are such that there is more often regurgitation of pancreatic juice into the bile duct, although reflux may occur in the opposite direction. This "pancreaticobiliary reflux" results in mutual stagnation of bile and pancreatic juice, and has a strong association with biliary carcinogenesis [15-17]. Carcinomas of the gallbladder and bile ducts are well described as a complication of PBM. The average age of patients with biliary tract cancer with PBM is ten years younger than those of cancer without PBM $[15,16]$. Treatment recommendations for PBM differ according to whether or not there is accompanying bile duct dilatation. In the former instance, prophylactic cholecystectomy with excision of the extrahepatic biliary tract with biliary reconstruction is the treatment of choice. ${ }^{(16)}$ Treatment of PBM without biliary dilatation, as in our patient, is controversial, with opinions split between prophylactic cholecystectomy only and more radical surgery [13]. The ERCP findings in our case showed a relatively 'normal' common channel length. However, the widened angle of the pancreaticobiliary junction was of concern and may have been contributory to the patient's unfavourable outcome.

Carcinosarcomas of the gallbladder, as in other organs, sometimes exhibit a polypoid configuration as in our case. The epithelial component is usually adenocarcinoma although squamous cell, adenosquamous, undifferentiated and small cell carcinomatous components have been reported. Sarcomatoid components may either be fibroblast-like or show evidence of heterologous differentiation including chondroid, rhabdomyoid and osteoid. Similar to their better documented pancreatic counterparts, some carcinosarcomas of the gallbladder may contain abundant giant cells that show typical morphologic and immunophenotypic features of osteoclastic cells $[1,2,5,14]$. Such cells were not seen in our case.

Our case was staged as a T1a, according to the AJCC/UICC staging for gallbladder carcinomas, there being no separate staging system for gallbladder carcinosarcomas or sarcomas [18]. There was no radiological evidence of nodal or liver involvement. The usual treatment of choice for gallbladder carcinosarcoma is surgery, and there have been no reports of successful treatment with radiotherapy or chemotherapy. The overall prognosis of malignant mixed tumours of the gallbladder is poor to dismal, with reported post-therapy survival times ranging from 0 to 85 months, with an average of 1.9 months and a median of 1.0 months in one series [1-14]. According to Zhang et al. [12] who performed a meta-analysis of 68 cases of carcinosarcoma of the gallbladder, tumor size over $5 \mathrm{~cm}$ was of signified a worse prognosis. There has been no significant difference in prognosis amongst patients with tumours showing chondroid, osteoid and rhabdomyosarcomatous differentiation [4]. Likewise, no significant differences in survival have been shown when comparing patients of different age groups 
Citation: Coetzee K, Omoshoro-Jones J, Michelow P (2011) Carcinosarcoma of the Gallbladder Arising in a Patient with Pancreaticobiliary Maljunction: A Case Report and Review of the Literature. J Cytol Histol 2:115. doi:10.4172/2157-7099.1000115

and genders, or when comparing those with cholelithiasis to those without. Whilst some reports state that curative resection may be effected if the tumor is detected whilst still confined to the gallbladder $[1,2,19,20]$, this was not the case in our patient. This may be attributable to the pathogenetic mechanisms underlying biliary carcinogenesis in PBM reflecting a "field effect", involving both the affected, dilated extrahepatic biliary tract and adjacent epithelium [14].

Carcinosarcomas are uncommon malignancies and can arise in almost any site where carcinomas arise. Some may have noticeable epithelial components but some may be morphologically indistinguishable from true sarcoma. In this patient, the primary tumor had malignant glands in addition to pleomorphic stromal cells. Without the history of a previous carcinosarcoma of the gallbladder, FNA of the sub-diaphragmatic mass after re-presentation of this patient would have yielded a wide differential diagnosis including true sarcoma, carcinosarcoma and melanoma. Ancillary investigations may be required in this regard. In an article by Lewis et al. [21], p63 proved particularly useful especially in head and neck carcinosarcomas and was positive in many cases where immunohistochemistry was negative for both pancytokeratin and epithelial membrane antigen.

In conclusion, we present the first case of carcinosarcoma of the gallbladder in a young patient with PBM. Her younger age at presentation and more aggressive clinical course may be related to the underlying PBM. This patient was too ill to undergo surgical biopsy of the recurrent mass but was able to tolerate FNA. FNA was able to document recurrent carcinosarcoma of the gallbladder, allowing appropriate management to be undertaken.

\section{Acknowledgements}

The authors thank Eric Liebenberg for his photographic assistance.

\section{References}

1. Agarwal T, Jain M, Goel A, Visayaragavan P, Gupta RK (2009) Carcinosarcoma of the gallbladder. Indian J Pathol Microbiol 52: 244-245.

2. Uzun MA, Koksal N, Gunerhan Y, Celik A, Gunes P (2009) Carcinosarcoma of the gallbladder: Report of a Case. Surg Today 39: 168-171

3. Born MW, Ramey WG, Ryan SF, Gordon PE (1984) Carcinosarcoma and Carcinoma of the gallbladder. Cancer 53: 2171-2177.

4. Ajiki T, Nakamura T, Fujino Y, Suzuki Y, Takeyama Y, et al. (2002) Carcinosarcoma of the gallbladder with chondroid differentiation. J Gastroenterol 37: 966-971.

5. Takahashi Y, Fukushima J, Fukusato T, Shiga J (2004) Sarcomatoid carcinoma with components of small cell carcinoma and undifferentiated carcinoma of the gallbladder. Pathol Int 54: 866-871.

6. Alvarez JA, Fernandez R, Aramburo J, Fradejas JM, Velasco A, et al. (1995) Carcinosarcoma of the gallbladder. Gastroenterol Hepatol 18: 81-83.

7. Lumsden AB, Mitchell WE, Vohman MD (1988) Carcinosarcoma of the gallbladder: a case report and review of the literature. Am Surg 54: 492-494.

8. Eriguchi N, Aoyagi S, Hara M, Hashino K, Imamura M, et al. (1999) A so-called carcinosarcoma of the gallbladder in a patient with multiple anomalies--a case report. Kurume Med J 46: 175-179.

9. Fagot H, Fabre JM, Ramos J, Laffay V, Guillon F, et al. (1994) Carcinosarcoma of the gallbladder: A case report and review of the literature. J Clin Gastroenterol 18: $314-316$

10. Inoshito S, Iwashita A, Enjoji M (1986) Carcinosarcoma of the gallbladder. Report of a case and review of the literature. Acta Pathol Jpn 36: 913-920.

11. Huguet KL, Hughes CB, Hewitt WR (2005) Gallbladder carcinosarcoma: a case report and literature review. J Gastrointest Surg 9: 818-821.

12. Zhang L, Chen Z, Fukuma M, Lee L, Wu M (2008) Prognostic significance of race and tumor size in carcinosarcoma of the gallbladder: a meta-analysis of 68 cases. Int J Clin Exp Pathol 1: 75-83.

13. Todani T, Arima E, Eto T, Funabiki T, Kakita A, et al. (1994) Diagnostic criteria of pancreaticobiliary maljunction. J Hepatobiliary Pancreat Surg 1: 219-221.

14. Al-Sheneber I, Jaber T, Huttner I, Arseneau J, Loutfi A (2002) Carcinosarcoma of the gallbladder: a case report and review of the literature. Saudi J Gastroenterol 8: 22-24.

15. Funabiki T, Matsubara T, Miyakawa S, Ishihara S (2009) Pancreaticobiliary maljunction and carcinogenesis to biliary and pancreatic malignancy. Langenbecks Arch Surg 394: 159-169.

16. Kamisawa T, Anjiki H, Eqawa N, Kurata M, Honda G, et al. (2008) Diagnosis and clinical implications of pancreaticobiliary reflux. World J Gastroenterol 14 6622-6626.

17. Kamisawa T, Kurata M, Honda G, Tsuruta K, Okamoto A (2009) Biliopancreatic reflux - pathophysiology and clinical implications. J Hepatobiliary Pancreat Surg 16: 19-24.

18. Greene FL, Page DL, Fleming DI (2009) AJCC Cancer Staging Manual. $7^{\text {th }}$ Ed New York: Springer-Verlag.

19. Adsay VN (2010) In Section VIII Alimentary tract and related organs, Chapte 38, Gallbladder, Extrahepatic Biliary Tree and Ampulla. In Sternberg's Diagnostic Surgical Pathology, $5^{\text {th }}$ Ed, Vol. 2. Mills SE, Carter D, Greenson JK, Reuter VE, Stoler MH. Baltimore, Lippincott Williams and Wilkins1626-1627.

20. Von Kuster LC, Cohen C (1982) Malignant mixed tumour of the gallbladder: report of two cases and a review of the literature. Cancer 50: 1166-1170.

21. Lewis JS, Ritter JH, El-Mofty S (2005) Alternative epithelial markers in sarcomatoid carcinoma of the head and neck, lung and bladder - p63, MOC-31 and TTF-1. Mod Pathol 18: 1471-1481. 